\title{
Time-frequency energy analysis of deepwater explosion shock wave signals based on HHT
}

\author{
Linna $\mathrm{Li}^{1}$, and Yue You ${ }^{1, *}$ \\ ${ }^{1}$ College of Science, Wuhan University of Science and Technology, 430065 Wuhan, China
}

\begin{abstract}
In order to study the time-frequency characteristics of shock wave signals under deep water explosion conditions, experiments are performed using water medium explosion containers to simulate different water depth conditions, and signal analysis is performed on the shock wave data obtained in the experiments. Traditional time-frequency analysis methods such as Fourier transform and wavelet transform have many limitations on deep-water explosion shock wave signal analysis, the HHT method is used to analyse the experimental data from the three-dimensional Hilbert spectrum, marginal spectrum and instantaneous energy spectrum. The results show that the time-frequency method can effectively extract the frequency components of the deep-water explosion load signal in different periods. It provides a reference for people to understand the time frequency characteristics of shock wave signals in deep water.
\end{abstract}

\section{Introduction}

With the rapid development of engineering explosion technology, explosion technology has been developed from the first military research to civil water explosion projects, such as channel widening, wharf construction and water conservancy facilities construction, etc. People are paying more and more attention to the safety and damage of engineering explosions Features [1]. Therefore, the research on underwater shock waves has important practical significance.

In the 1820 s, Fourier transform was widely used in the field of signal processing as a classic signal analysis technique after Fourier put forward the "Analytical Theory of Heat Conduction" [2]. It can transform the time series samples in the time domain into the spectrum in the frequency domain, but it cannot reflect the changes of local instantaneous frequency, so it is only suitable for quasi-steady-state signals.

At present, the most widely used tool for processing nonstationary signals is wavelet analysis, which was introduced by French physicist Morley in the 1980s [3]. Wavelet transform uses wavelet windows to solve the contradiction of time frequency resolution, which is obtained in blasting signal analysis. It has a wide range of applications. Literature [4] uses wavelet packet technology to analyse the energy propagation law of blasting signals under different blasting conditions, Hubei Provincial Key Laboratory Fund of Metallurgical Industry Process System Science. And deepens people's understanding of the harm of

\footnotetext{
* Corresponding author: 2591107497@qqq.com
} 
blasting vibration mechanism; However, the diversity of wavelet bases leads to problems in the choice of wavelet bases.

Hilbert-Huang signal processing method [5] was proposed by Chinese American N.E. Huang et al. in 1988. HHT is an adaptive transformation method that does not need to choose a wavelet basis, and is suitable for analysing non-stationary signals [6]. This method breaks the limit of the uncertainty principle and shows better resolution in time-rate analysis. It has been applied to many fields. Therefore, this paper uses the HHT method to analyse the shock wave signal of deep water explosion, observe the change of the shock wave signal's frequency with time and learn the time frequency energy distribution the characteristics of the shock wave signal in the deep water environment.

\section{Introduction to HHT principle}

\subsection{EMD decomposition}

In the stepwise decomposition of the EMD method, the intrinsic modal function must meet two conditions: (1) In the entire data sequence, the number of signal extreme points and zerocrossing points are the same or differ by one; (2) The local signal at any point in time The maximum and minimum points are fitted to obtain a set of upper and lower envelopes, the local mean of which is zero, which means that the upper and lower envelopes are locally symmetric about the time axis. Any complex signal can be decomposed into the sum of $n$ IMF components. Specific steps are as follows:

First, find all the maximum and minimum values of the original signal $x(t)$, and use cubic spline interpolation to fit the local maximum and minimum to draw the upper and lower envelopes. And calculate the mean envelope $\mathrm{ml}(\mathrm{t})$ of the upper and lower envelopes. Subtract $\mathrm{ml}(\mathrm{t})$ from the original sequence $\mathrm{x}(\mathrm{t})$ to get the new sequence $\mathrm{hl}(\mathrm{t})$ :

$$
x(t)=m 1(t)-h 1(t)
$$

Then, judge whether this $\mathrm{h} 1(\mathrm{t})$ satisfies the two conditions of the intrinsic modal function. If the conditions are met, the first IMF component is decomposed; if not, based on the signal $\mathrm{h} 1(\mathrm{t})$, repeat the above process $\mathrm{n}$ times, until the condition is met. The acquisition of IMF components usually requires several iterations.

Through the above process, the first IMF component is obtained as $\mathrm{c} 1(\mathrm{t})$, and then $\mathrm{c} 1(\mathrm{t})$ is separated to obtain the difference signal $\mathrm{r} 1(\mathrm{t})$ :

$$
\mathrm{r} 1(\mathrm{t})=\mathrm{x}(\mathrm{t})-\mathrm{c} 1(\mathrm{t})
$$

Finally, take r1(t) as a new data sequence, repeat the above process to get the second component $\mathrm{c} 2(\mathrm{t})$, repeat $\mathrm{n}$ times until the end of the loop.

When the residual $r_{n}$ becomes a monotonic function and no IMF component can be decomposed, the decomposition process of EMD is terminated. The original signal $x(t)$ is equivalent to the combination of many IMF components and a residual term $r_{n}(\mathrm{t})$,

$$
\sum_{i=1}^{n} c_{i}(t)+r_{n}(t)
$$

\subsection{Hilbert transform}

The Hilbert transform transforms the IMF components to obtain the instantaneous frequency. The sum of the instantaneous frequencies of all IMF components is the Hilbert spectrum. 
Defined $a_{j}(t)$ as the instantaneous amplitude, and $e^{\theta_{j}}(t)$ is the instantaneous phase,

$$
h(\omega, t)=\operatorname{Re} \sum_{j=1}^{n} a_{j}(t) e^{\theta_{j}}(t)
$$

The instantaneous frequency is $\omega_{j}(t) \frac{d \theta_{j}(t)}{d t}$. Integrate the Hilbert spectrum over time to obtain the marginal spectrum:

$$
h(\omega)=\int_{-\infty}^{\infty} h(\omega, t) d t
$$

Finally, the square of the amplitude is integrated with the frequency, and the instantaneous energy spectrum is defined by the above marginal spectrum:

$$
E(t)=\int_{\omega}^{\infty} H^{2}(\omega, t) d t
$$

\section{Deep water explosion experiment}

$10 \mathrm{~g}$ TNT equivalent can simulate a cylindrical underwater explosion container with a water depth of $200 \mathrm{~m}$. The test conditions are as follows: the mass of the TNT charge is $1 \mathrm{~g}$, the distance between the measuring point and the explosive is $0.25 \mathrm{~m}$, and the explosive and the measuring point are at the same water depth, $0.5 \mathrm{Mpa}$ simulates a water depth of $50 \mathrm{~m}$.

\section{EMD decomposition and signal reconstruction}

The underwater explosion shock wave signal is decomposed by EMD, and the reconstructed signal and the error distribution diagram between the reconstructed signal and the measured signal are obtained.

It can be seen from Figure 1 and Figure 2 that the relative error between the reconstructed signal obtained by EDM decomposition and the measured signal is only about $10^{-15}$, and it has a high degree of consistency. The EMD method reflects the signal characteristics with high accuracy and is suitable for signal analysis of deep water explosion shock waves.

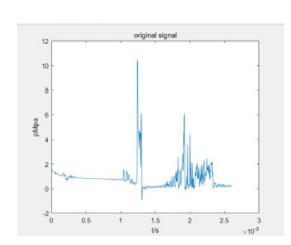

Fig. 1. Original signal.

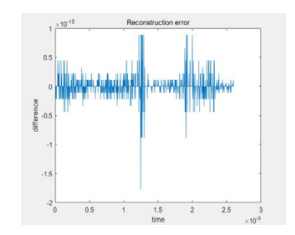

Fig. 2. Reconstruction error.

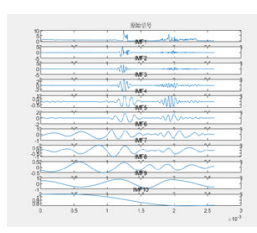

Fig. 3. IMF component of the original signal.

The original signal is decomposed into 10 IMF components and 1 margin, and decomposed from high frequency to low frequency according to the time scale. Each IMF component displays the characteristics of the original signal with a different resolution. Among them, IMF1-IMF3 belong to the high-frequency part and occupy less energy; IMF4IMF6 are the dominant frequency bands of the original signal, and the corresponding amplitude is larger, which is the concentrated frequency band of signal energy; IMF7-IMF10 are the low frequency part of the shock wave signal; The last margin $r$ represents the average trend of the signal, and the amplitude is small. It can be seen from this that EMD 
decomposition does not need to set any restrictions, and the original signal can be decomposed into a limited number of IMF components according to the characteristics of the signal itself, there will be no omissions or losses, and the adaptability and reconstruction are strong.

\section{Quantitative analysis}

Through the HHT method, we have obtained the time domain diagram and instantaneous energy spectrum of different hydraulic shock wave loads under the same dosage as shown in Figure 6 and Figure 7. It can be seen from the comparison of the two figures that the threedimensional Hilbert spectrum clearly reflects the transient characteristics of the shock wave signal. You can see the distribution of the energy of the signal over time and frequency, more comprehensively showing the corresponding relationship between the energy and the fluctuation time history, and it has good consistency with the original signal time domain diagram. The four peaks appearing under the four water pressures are roughly $0.8 \mathrm{~ms}, 1.25 \mathrm{~ms}$, $2 \mathrm{~ms}$, and $2 \mathrm{~ms}$. The appearance time of the maximum amplitude of the original signal just corresponds to the energy peak of the instantaneous energy spectrum. It shows that this method has advantages in studying the energy distribution of shock wave signal in time. The four pictures of the three groups of experiments indicate that the water pressure environment is at $0 \mathrm{mpa}, 0.5 \mathrm{mpa}, 1 \mathrm{mpa}$, and $2 \mathrm{mpa}$.
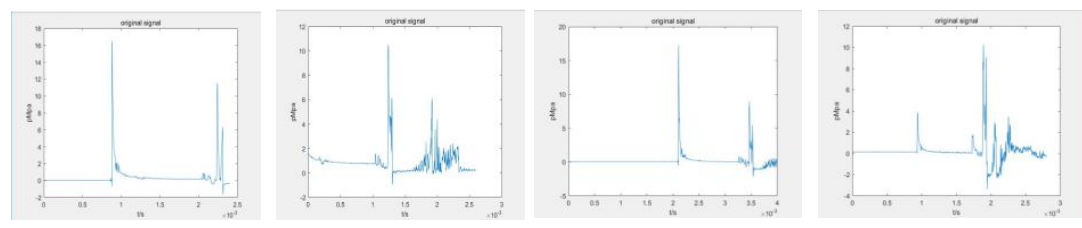

Fig. 4. Time domain diagram of shock wave load pressure signal.
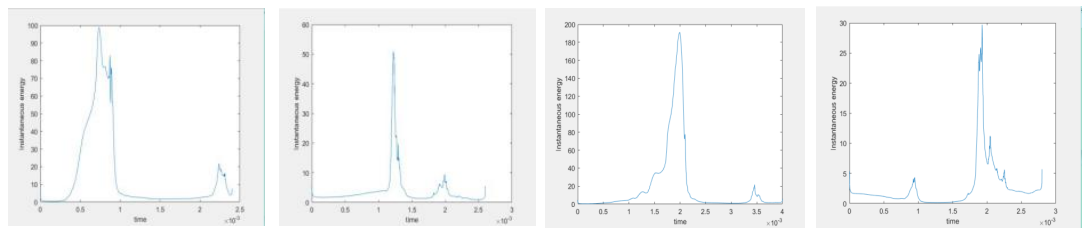

Fig. 5. Instantaneous energy spectrum.
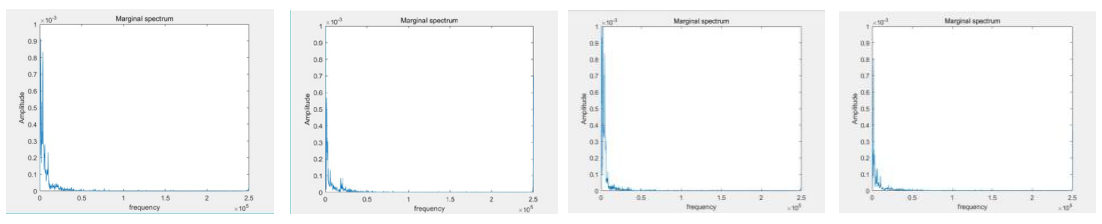

Fig. 6. Marginal spectrum.

The Hilbert energy spectrum more intuitively shows the accumulation of energy at different instantaneous frequencies over time. We processed the experimental data to obtain the instantaneous energy spectra of the shock wave load signals measured by four different hydraulic pressures. By observing the energy spectra, the relative energy of each frequency band can be obtained more accurately. It is found by comparison that under the same dose and distance, as the water pressure deepens, the shock wave reaches the sensor and quickly reaches its peak value. The water depth shown in the figure has little effect on the shock wave 
peak value. Observation from the marginal spectrum in Figure 8 shows that as time goes by, the energy is gradually attenuating and tends to develop towards low frequencies. It shows that not only the pressure peak is caused by the shock wave damage, but the low frequency band will also affect the structure. The dominant frequency of shock wave signal is mainly concentrated within $50 \mathrm{khz}$, especially the low frequency energy below $20 \mathrm{khz}$ is the largest.

\section{Conclusion}

Analyse the measured underwater explosion shock wave signal based on the HHT method. From the Hilbert energy spectrum and the marginal spectrum analysis, it is found that the dominant frequency of the shock wave signal is mainly concentrated within 50khz, especially the low frequency energy below $20 \mathrm{khz}$ is the largest, and they are all concentrated in the low frequency part, which is basically consistent with the empirical theoretical value. In terms of signal analysis, the HHT method shows strong resolution and recognition capabilities.

Experiment comparison found that under the same dose and distance, as the water pressure deepened, the shock wave reached the sensor and reached the peak quickly, indicating that the water depth had no obvious influence on the shock wave peak.

\section{References}

1. Q. Yin. The Status and Trends of Underwater Explosion Research, ST, pp. 8-14 (2003)

2. X. Li.Extraction of micro seismic wave forms characteristics prior to rock burst using Hilbert-Huang transform, M, 91 (2016)

3. H. Jia.Research on Time-Frequency Characteristics of Underwater Explosion Signal of Metal Detonating Cord Based on HHT, EB, 21, pp. 5-8 (2015)

4. T. Ling, X. Li, T.H. Ling, X.B.Li.The Energy Distribution Law of Blasting Vibration Signals in Different Frequency Bands, JCSU (Natural Science Edition), pp. 310-315 (2004)

5. L. Cheng, J. Wang,L. Cheng and J.Wang. HHT-based analysis of the characteristics of underwater explosion shock wave signals, SW, 16, pp. 25-27( 2010)

6. S. Hui, Q.R.Si, C. Ning, Y. Shouqi.HHT-based feature extraction of pump operation instability under cavitation conditions through motor current signal analysis, M. Syst. S.P, 139, (2020)

7. W.Wu, J.Wu. Non-stationary signal analysis based on HHT and machine learning, UESTC, (2019) 